\title{
Atomic Hydrogen at High Redshift
}

\author{
F.H. Briggs \\ Kapteyn Astronomical Institute, Postbus 800, 9700 AV Groningen, The \\ Netherlands
}

\begin{abstract}
After the production of the ionizing background by the first generation of stars, neutral gas must be confined to sufficiently high density to be self-shielding and remain neutral. Neutral gas is an identifier of the presence of confining gravitational potentials and a tracer of the kinematics of the potential. Kinematical studies are being extended to neutral atomic gas at high redshift.
\end{abstract}

\section{Introduction}

This discussion emphasizes three principal areas: the nature of galaxies selected for neutral gas content, the role of $\mathrm{HI}$ as a tracer of galactic potentials, and the "highest redshift HI" that constitutes the IGM during between the Epoch of Recombination and the period of reionization that is driven by the first generation of luminous objects.

The global neutral gas content of the Universe as a function of time shown in Figure 1 provides a framework for the discussion. This diagram begins at the recombination epoch at $z \sim 1000$ and continues to the present. The "Dark Age" (Rees 1996, 1999), during which the cosmic background radiation propagates freely through an IGM of neutral baryons, is ended when the first stars or luminous compact $\mathrm{AGN}$-like objects generate the ionizing background radiation that is responsible for ionizing the bulk of the Universe's baryons. There has been a remarkable convergence toward agreement of empirical evidence on the number of baryons through the measurement of light elements combined with Big Bang Nucleosynthesis models (Tytler et al. 2000) and through measurements of the CMB fluctuation spectrum (Hu et al. 2000). This fixes the cosmological density of neutral gas through the Dark Age. Just when and how the Dark Age ends is the subject of great current interest for both theorists and observers.

\section{Nature of HI selected galaxies}

Through the period of galaxy assembly and most vigorous star formation, we monitor the neutral gas content of the Universe through the damped Lyman $-\alpha$ DLA absorption line seen against the continuum of quasars. At very low redshift, we determine the HI content and general distribution of neutral gas through blind and targeted $21 \mathrm{~cm}$ line observations of nearby galaxies. Figure 1 shows a marked drop in $\Omega_{\text {gas }}$ between DLA points and the $21 \mathrm{~cm}$ values, leading to the concern that the HI content of the nearby Universe might be severely underesti- 


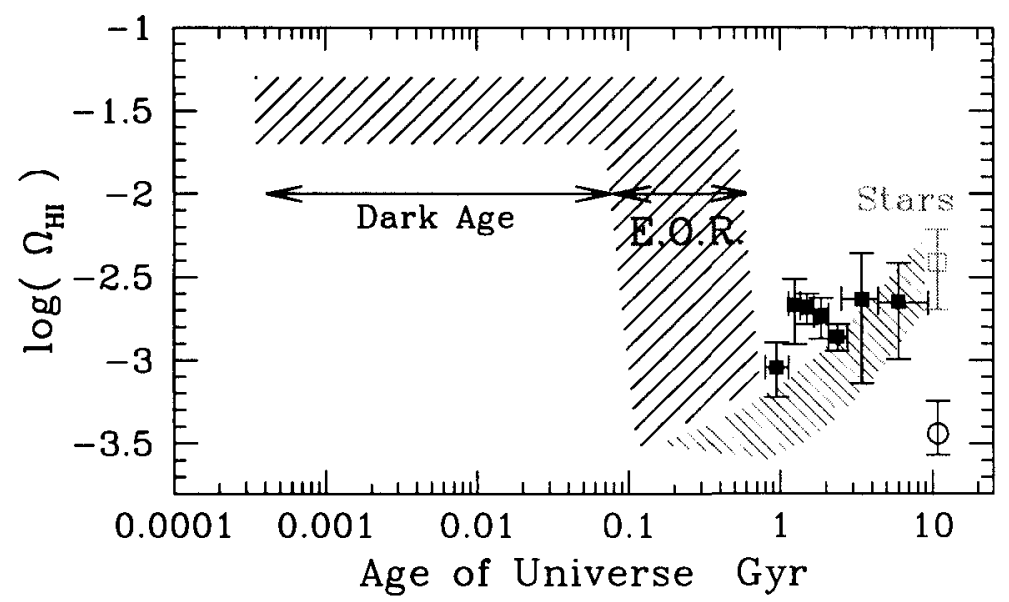

Figure 1. Cosmological density of neutral gas, $\Omega_{\text {gas }}$, as a function of the age of the Universe since the Recombination Epoch. The neutral gas content during the Dark Age is equal to the baryonic mass density. The timing of the Epoch of Reionization EOR is not yet well determined, falling somewhere in the redshift range 5 to 30 . The filled square data points come from Damped Lyman $-\alpha$ quasar absorption-line statistics (Wolfe et al. 1986, Rao \& Turnshek 2000), and the open circle at the present epoch results from surveys in the $21 \mathrm{~cm}$ line. A light shaded area is drawn to represent the rise in the baryonic content of stellar populations after the EOR, until reaching the measured value at present (open square).

mated or hampered by incompleteness. This is not the case - a series of "blind" HI surveys of significant volumes of sky (Zwaan et al. 1997, Spitzak \& Schneider 1998, Kilborn et al. 1999, Rosenberg \& Schneider 2000) now show that HI clouds reside either in the galaxies disks, where they exhibit galactic revolution, or they sit in the halos of galaxies, as tidal debris, streams, small clouds or dwarf galaxies. There are no known cases of "free floating" intergalactic clouds not associated with a star-bearing galaxy or galaxy group.

The astrophysics of neutral gas clouds and the absence of intergalactic specimens is probably quite simple: clouds must be confined to sufficiently high density that they can remain neutral in the face of an ionizing background radiation field. Clouds that are not confined become ionized and join the bulk of the Universe's baryons that constitute the intergalactic medium (Shull et al. 1999). The gravitational potentials provided by galaxy halos are natural confinement vessels for hydrogen, but once confined to adequate density to become self-shielding, the gas is also vulnerable to the instabilities that give rise to star formation. There appears to be an inevitable association of HI with stars in the nearby Universe (Zwaan 2000).

A net result of the $21 \mathrm{~cm}$ line observations is that there has been a convergence on a value for the integral HI content of the local Universe, both from the blind HI surveys (Zwaan et al. 1997) and from $21 \mathrm{~cm}$ line observations that target optically selected galaxies (Fall \& Pei 1989, Rao \& Briggs 1993). 
At high redshift, HI-rich objects are selected through damped Lyman $-\alpha$ absorption (Wolfe et al. 1986). While much progress is being made in measuring the evolution of metal content in these neutral clouds (Pettini et al. 1997, 2000; Prochaska \& Wolfe 2000), the identification of the galaxies that host the HI clouds has been extremely difficult (see for example Ellison et al. 2000), even at redshifts less than one (Steidel et al. 1994, Rao \& Turnshek 1998), causing basic questions about the morphologies of the hosts to remain unanswered.

Somerville (2000) has recently reviewed the controversial hypotheses about the nature of DLA absorbers. Opinions range from large, well-formed disk-like systems (Prochaska \& Wolfe 1997, 1998) to a collection of small objects in midmerge (Haehnelt, Steinmetz \& Rauch 1998). At both extremes, the authors comment that the DLAs are likely to be the "best probe of the progenitors of the normal present-day galaxies."

The DLA systems are selected for their HI absorption cross section rather than optical brightness, and it is likely to require gas-sensitive observational techniques to determine their nature and relation to present day galaxies. A direct measurement of the HI content of these high redshift galaxies at $z \sim 3$ to 4 will be possible with a next-generation radio telescope such as the Square Kilometer Telescope SKA (van Haarlem 1999). Current telescopes, including Westerbork, VLA and the new GMRT in India, will be effective to redshifts $z \sim 0.3$.

\section{Prospects for kinematic studies in $\mathrm{HI}$ at high $\mathrm{z}$}

High spectral resolution kinematic studies of the low-ionization metal lines formed in the DLA gas layers have been central to the controversy over the nature of the DLAs. As in the $21 \mathrm{~cm}$ line synthesis mapping of galaxies that led to the measurement of dark matter content and distribution in spiral galaxies in the nearby universe, the large velocity spreads observed for the metal lines may be indicative of gas motions in massive potentials.

Since neutral gas must be confined to be self-shielding to the ionizing background, it is likely to be the optimal kinematical tracer. Tidal interactions that disperse gas or supernova ejections that shock it, will lead to ionization and disappearance from detectability to $\mathrm{HI}$ sensitive probes. Conventional $21 \mathrm{~cm}$ line mapping observations could be extended to redshifts $\sim 1$ with SKA, but mapping the detailed kinematics of $21 \mathrm{~cm}$ emission from $z>1$ galaxies is likely to remain out of reach.

There are good prospects, however, for mapping HI in absorption against extended background radio sources with existing instrumentation. An example of how this might work is shown by the observation of 3C196 by Briggs, de Bruyn and Vermeulen (see Briggs 1999).

\section{Epoch of reionization}

The Dark Age between the epoch of recombination and the epoch of reionization is also the "great epoch of neutrality". There has been a recent surge of theoretical interest in the first generation of stars and their effect on the neutral IGM. Little is yet known observationally, and there are only loose constraints on 
the redshift range over which reionization occurs: Numerical simulations favor $z_{\text {eor }}$ from 8 to 20 . $z_{\text {eor }}$ cannot be more recently than $z \sim 5.5$ since UV continuum can be detected against the highest redshift QSOs (Zheng et al. 2000); $z_{\text {eor }}$ cannot be greater than 30 without causing observable suppression of the first peak in the power spectrum of anisotropies in the CMB (de Bernardis et al. 2000). Two recent papers (Shaver et al. 1999, Tozzi et al. 2000) address the possibilities for direct radio observation of the neutral gas through this period when the first stars form and the seed of galactic structures emerge. This Epoch of Reionization is one of the next frontiers of observational cosmology.

\section{References}

Briggs. F.H. 1999, in Perspectives on Radio Astronomy: Science with Large Antenna Arrays, van Haarlem, M. P. ed. (ASTRON: Dwingeloo) p 75 (astro-ph/9910415)

de Bernardis, et al. 2000, Nature, 404, 955

Ellison, S.L. et al. 2000, ApJ, in press (astro-ph/0010427)

Fall, S.M. \& Pei, Y.C. 1989, ApJ, 337, 7

Haehnelt, M.G., Steinmetz, M. \& Rauch, M. 1998, ApJ, 495, 647

Hu, W., Fukugita, M., Zaldarriaga, M. \& Tegmark, M. 2000 (astro-ph/0006436)

Kilborn, V.A., Webster, R.L. \& Stavely-Smith, L. 1999, PASA, 16, 8

Pettini, M. et al. 1997, ApJ, 486, 665

Pettini, M. et al. 2000, ApJ, 532, 65

Prochaska, J.X. \& Wolfe, A.M. 1997, ApJ, 487, 73

Prochaska, J.X. \& Wolfe, A.M. 1998, ApJ, 507, 113

Prochaska, J.X. \& Wolfe, A.M. 2000, ApJ, 533, L5

Rao, S.M. \& Briggs, F.H. 1993, ApJ, 454, 69

Rao, S.M. \& Turnshek, D.A. 1998, ApJ, 500, L115

Rao, S.M. \& Turnshek, D.A. 2000, ApJS, in press (astro-ph/9909164)

Rees, M. J. 1996 (astro-ph/9608196)

Rees, M. J. 1999 (astro-ph/9912345)

Rosenberg, J.L. \& Schneider, S.E. 2000 (astro-ph/0010376)

Shaver, P.A. et al. 1999, A\&A, 345, 380

Shull, J.M., Penton, S.V. \& Stocke, J.T. 1999, PASA, 16, 95

Somerville, R.S. 2000, in Galaxy Disks and Disk Galaxies, eds. J.G. Fumes and E.M. Corsini (astro-ph/0010350)

Spitzak, J.G. \& Schneider, S.E. 1998, ApJS, 119, 159

Steidel, C.C. et al. 1994, AJ, 108, 2046

Tozzi, P. et al. 2000, ApJ, 528, 597

Tytler, D., O'Meara, J.M., Suzuki, N. \& Lubin, D. 2000, PhST, 85, 12 (astro$\mathrm{ph} / 0001318)$

van Haarlem, M. P. 1999, Perspectives on Radio Astronomy: Science with Large Antenna Arrays, (ASTRON: Dwingeloo, The Netherlands). 
Wolfe, A.M., Turnshek, D.A., Smith, H.E. \& Cohen, R.D. 1986, ApJS, 61, 249 Zheng, W. et al. 2000, AJ, 120, 1607

Zwaan, M.A., Briggs, F.H., Sprayberry, D. \& Sorar, E. 1997, ApJ, 490, 173

Zwaan, M.A. 2000, PhD Thesis, University of Groningen 